Supporting Information

\title{
A Multifunctional Self-Powered Switch toward Delay-Characteristic Sensors
}

Haoyu Wang ${ }^{\mathrm{a}, \mathrm{b}}$, Jiaqi Wang ${ }^{\mathrm{a}, \mathrm{b}}$, Xin Xia ${ }^{\mathrm{a}}$, Dong Guan ${ }^{\mathrm{a}}$, Yunlong Zi $\mathrm{i}^{\mathrm{a}, \mathrm{b},{ }^{*}}$

a. Department of Mechanical and Automation Engineering, The Chinese University of Hong Kong, Shatin, N.T., Hong Kong, China

b. Shun Hing Institute of Advanced Engineering, The Chinese University of Hong Kong,

Shatin, N.T., Hong Kong, China

*Corresponding author, email: ylzi@cuhk.edu.hk

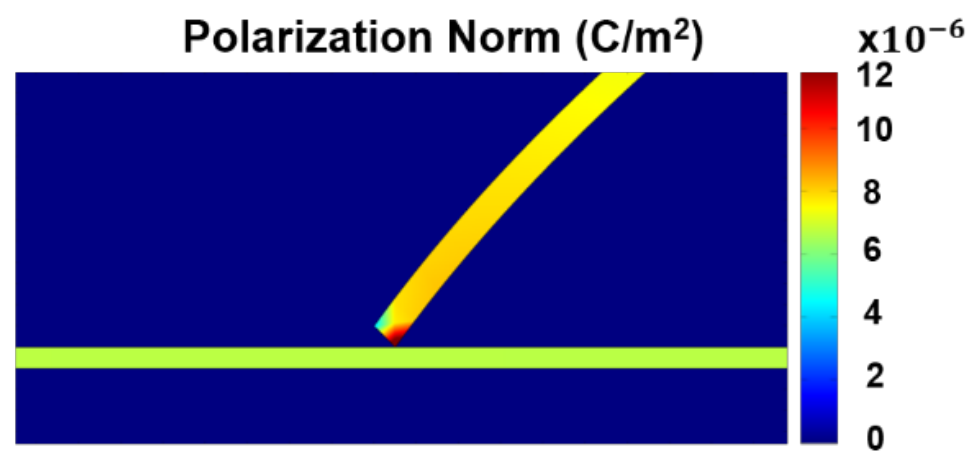

Figure S1. The simulated polarization norm distribution of EBR film in Status 2. The bottom surface polarization charge density of the free end of the NBR film could be higher than the top surface. 


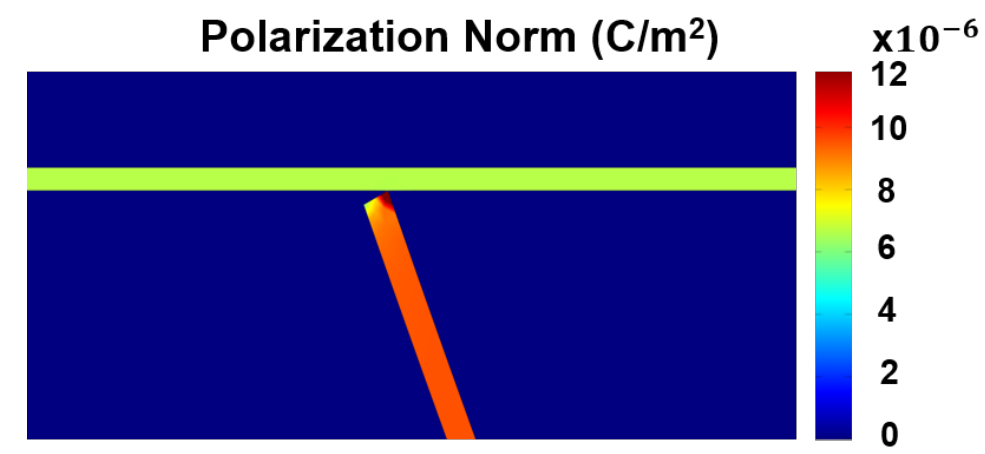

Figure S2. The simulated polarization norm distribution of NBR film when it reaches the top copper layer.

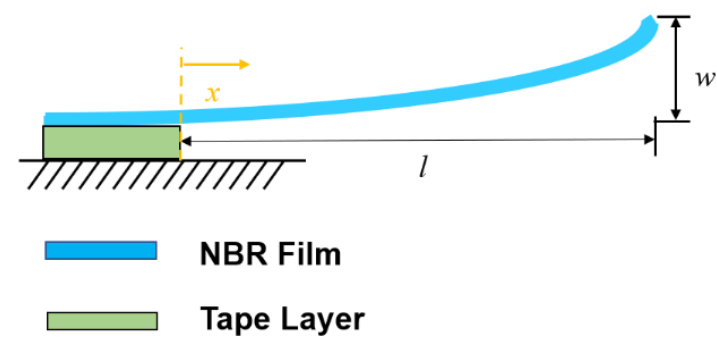

Figure S3. The equilibrium condition of the EFOS.

Note S1. The calculation method of the surface charge density of the NBR film.

The polarization charge density on the NBR surface can be calculated by

$$
\begin{aligned}
& \vec{P}=\chi_{e} \varepsilon_{0} \vec{E} \\
& \sigma_{P}=-\overrightarrow{e_{n}} \cdot\left(\overrightarrow{P_{2}}-\overrightarrow{P_{1}}\right)
\end{aligned}
$$

where the polarization vector, electric susceptibility, vacuum permittivity, electric field, bound surface charge density and normal unit vector from medium 1 to medium 2 on the interface can be denoted as $\vec{P}, \chi_{e}, \varepsilon_{0}, \vec{E}, \sigma_{P}$ and $\overrightarrow{e_{n}}$ respectively. Here, the medium 1 is the NBR film, and the medium 2 is the air. 
Note S2. The EFOS's critical equilibrium condition.

The equilibrium condition of the EFS when the NBR film contact with the top copper layer, as descripted in

$$
\overrightarrow{F_{e}}=-\vec{F}_{s}
$$

where the elastic force $F_{\mathrm{e}}$ is balanced with the net electrostatic force $F_{\mathrm{s}}$.

Let's consider the $F_{e}$ which was integrated by the distributed load $q_{e}$ as determined by the NBR film's Young's modulus $Y$, moment of inertia $I$ and length $l$.

$$
\overrightarrow{F_{e}}=\int \overrightarrow{q_{e}}(x) d x
$$

When the generated deformation is $w$, as shown in Figure S3, $q_{e}$ at each position can be calculated by equation

$$
q_{e}(x)=\frac{24 Y \operatorname{II}(x)}{x^{2}\left(6 l^{2}-4 l x+x^{2}\right)}=\frac{2 a h^{3} Y w(x)}{x^{2}\left(6 l^{2}-4 l x+x^{2}\right)}
$$

where $a$ present the width of the NBR film and $h$ present the thickness of the NBR film.

Then, let's consider the $F_{\mathrm{s}}$ as integrated from the product of the electric field and the bound surface charge density denoted as $\vec{E}$ and $\sigma_{P}$, respectively:

$$
\overrightarrow{F_{s}}=\int \sigma_{P} \vec{E} d S
$$

The $\sigma_{P}$ can be calculated by

$$
\sigma_{P}=-\overrightarrow{e_{n}} \cdot\left(\overrightarrow{P_{2}}-\overrightarrow{P_{1}}\right)=\overrightarrow{e_{n}} \cdot \overrightarrow{P_{1}}=\overrightarrow{e_{n}} \cdot \chi_{e} \varepsilon_{0} \vec{E}
$$

where the polarization vector, the electric susceptibility and vacuum permittivity can 
be denoted as $\vec{P}, \chi_{e}$ and $\varepsilon_{0}$, respectively. Here we ignore the influence of air. Then the net surface charge density can be calculated by

$$
\sigma_{n}=\sigma_{p u}+\sigma_{p b}=\left(\overrightarrow{e_{n 1}}+\overrightarrow{e_{n 2}}\right) \cdot \chi_{e} \varepsilon_{0} \vec{E}
$$

where the upper surface charge density of NBR film, bottom surface charge density of NBR film, the normal unit vector from NBR upper surface to air on the interface and the normal unit vector from NBR bottom surface to air on the interface can be denoted as $\sigma_{p u}, \sigma_{p b}, \overrightarrow{e_{n 1}}$ and $\overrightarrow{e_{n 2}}$, respectively. The electric field is generated by FS-TENG. Assume for each movement of FS-TENG, the transferred charge, the area of the copper layer of the EFOS and the distance between the two copper layers can be denoted as $Q$, $S$ and $d$, respectively. The strength of the electric field can be calculated by equation

$$
\vec{E}=\frac{U}{d} \overrightarrow{e_{E}}=\frac{Q}{C d} \overrightarrow{e_{E}}=\frac{Q}{\varepsilon S} \overrightarrow{e_{E}}
$$

where $\varepsilon$ presents dielectric permittivity. Based on equation (4)-(9), equation (3) can be adjusted to equation (10), which describes the boundary equilibrium condition of the EFS.

$$
\int \frac{2 a h^{3} Y w(x)}{x^{2}\left(6 l^{2}-4 l x+x^{2}\right)} d x=\int\left[\left(\overrightarrow{e_{n 1}}+\overrightarrow{e_{n 2}}\right) \cdot \chi_{e} \varepsilon_{0} \frac{Q}{\varepsilon S} \overrightarrow{e_{E}}\right] \frac{Q}{\varepsilon S} a d x
$$

As the EFOS and TENG scaled down, let's using scaling law to analyze the boundary equilibrium condition. The elastic force $F_{e}$ catch a relationship with the linear dimension $l$

$$
F_{e} \propto\left[l^{2}\right]
$$

where the $a, h, w, x$ and $l$ have the proportional relationship with the size of the EFOS. 
As for the electrostatic force $F_{s}$, it has the positive relationship with the $E$. Let's keep the value $Q / S$ unchanged as the charge density keeps identical, which makes the $E$ is unchanged. Then the electrostatic force is unchanged. Based on equation (9), the $Q$ has proportional relationship with the area size $S$, and thus when we proportionally scale down both the EFOS and FS-TENG in the same rate, the $E$ is not changed. Then the electrostatic force is determined by the $a d x$, which gives the relationship between the $F_{s}$ and the linear dimension $l$

$$
F_{s} \propto\left[l^{2}\right]
$$

Let's reconsider the equation (10), by scaling down the size of the EFOS and the FSTENG, the elastic force and electrostatic force will reduce in the same rate, which means boundary equilibrium condition always keep in balance. Then, this theory demonstrates the possibility to scale down/up the device, enabling optimization of the size.

At the same time, the EFOS may not be scaled down indefinitely. With the scaling down of the EFOS, the edge effect from other forces such as surface tension may make great influence to the system.

Video S1. A successful movement circle of EFOS (2.5 times slower)

Video S2. An unsuccessful movement circle of EFOS for not enough voltage (2.5 times slower) 
Video S3. The repeatability experiment of the EFOS

Video S4. Self-powered EFOS wireless sensing system in delay characteristic

Video S5. Self-powered EFOS wireless sensing system in quick movement of TENG

Video S6. Self-powered EFOS optical coding communication system

Video S7. Self-powered CEFES mechanical motion sensing system in delay characteristic 